\title{
An approach to output-feedback MPC of stochastic linear discrete-time systems
}

\author{
Marcello Farina, ${ }^{\mathrm{a}}$ Luca Giulioni, ${ }^{\mathrm{a}}$ Lalo Magni, ${ }^{\mathrm{b}}$ and Riccardo Scattolini ${ }^{\mathrm{a}}$ \\ ${ }^{a}$ Dipartimento di Elettronica, Informazione e Bioingegneria, Politecnico di Milano, Milan, Italy: \\ \{marcello.farina,luca.giulioni,riccardo.scattolini\}@polimi.it

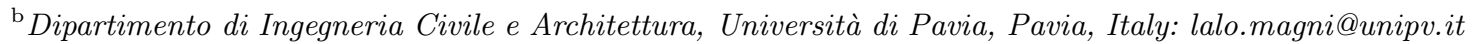

\begin{abstract}
In this paper we propose an output-feedback Model Predictive Control (MPC) algorithm for linear discrete-time systems affected by a possibly unbounded additive noise and subject to probabilistic constraints. In case the noise distribution is unknown, the probabilistic constraints on the input and state variables are reformulated by means of the Chebyshev - Cantelli inequality. The recursive feasibility is guaranteed, the convergence of the state to a suitable neighbor of the origin is proved under mild assumptions, and the implementation issues are thoroughly addressed. Two examples are discussed in details, with the aim of providing an insight into the performance achievable by the proposed control scheme.
\end{abstract}

\section{Introduction}

The problem of designing robust deterministic Model Predictive Control (MPC) schemes has nowadays many solutions, see for example $[30,18]$. However, the available approaches are in general computationally very demanding, since they either require the solution to difficult on-line min-max optimization problems, see [20], or the off-line computation of polytopic robust positive invariant sets, see [24]. In addition they are conservative, mainly because they (implicitly or explicitly) rely on worst-case approaches.

If the uncertainties or the state and control disturbances are characterized as stochastic processes, constraints should be reformulated in a probabilistic framework $[32,14]$. However, worst-case deterministic methods do not take advantage of the available knowledge on the characteristics of the process noise, such as their probability density function, and cannot even guarantee recursive feasibility in case of possibly unbounded disturbances.

Starting from the pioneering papers $[31,17]$, these reasons have motivated the development of MPC algorithms for systems affected by stochastic noise and subject to probabilistic state and/or input constraints. Mainly two classes of algorithms have been developed so far. The first one relies on the randomized, or scenario-based approach, see e.g., [4,3], a very general methodology that allows to consider linear or nonlinear systems affected by noise with general distributions characterized by possibly unbounded and non-convex support. As a main drawback, randomized methods are still computationally very demanding for practical implementations and their feasibility and convergence properties are difficult to prove.

The second approach, referred in [34] as probabilistic approximation method, is based on the point-wise reformulation of probabilistic, or expectation, constraints in deterministic terms to be included in the MPC formulation. Interesting intermediate methods have been proposed in [1], where a finite number of disturbance realizations are assumed, and in [15], where constraints averaged on time are considered. Among the probabilistic approximation algorithms, a further distinction can be based on the noise support assumptions, which can be either bounded, e.g., as in $[15,16,5]$ or unbounded, see $[33,28,13,34,6,27]$. While for bounded disturbances recursive feasibility and convergence can be established, the more general case of unbounded noise poses more difficulties and some specific solutions and reformulations of these properties have been adopted; for example in [6] the concept of invariance with probability $p$ is used, while in [27] the definition of probabilistic resolvability is introduced. Also, linear systems with known state have generally been considered, with the notable exceptions of $[33,13,5]$, where output feedback methods have been proposed.

Finally, it must be remarked that some of the mentioned approaches have been successfully applied in many applicative settings, such as building temperature regulation [26] and automotive applications [12,3,2]. In this paper, an output feedback algorithm for linear 
discrete-time systems affected by a possibly unbounded additive noise is proposed, based on the preliminary work in $[8,9]$. If the noise distribution is unknown, the probabilistic constraints on the inputs and state variables are reformulated by means of the Chebyshev Cantelli inequality [22]. This idea has been originally proposed in [19] for the design of decentralized controllers and in [21] in the context of MPC, and has been later used in $[34,12]$. With respect to $[8,9]$, in this paper we address also the case when the noise distribution is known (i.e., Gaussian), we examine in depth the implementation aspects, we propose two novel and theoretically well funded approximated schemes, and we provide full implementation details. In particular, we show that the algorithm computational load can be made similar to the one of a standard stabilizing MPC algorithm with a proper choice of the design parameters (e.g., converting all constraints to linear matrix inequalities, LMIs), so that the application of the proposed approach to medium/large-scale problems is allowed. The recursive feasibility of the algorithm is guaranteed by a switching MPC strategy which does not require any relaxation technique, and the convergence of the state to a suitable neighbor of the origin is proved.

The paper is organized as follows. In Section 2 we first introduce the main control problem, then we define and properly reformulate the probabilistic constraints. In Section 3 we formulate the Stochastic MPC optimization problem and we give the general theoretical results. Section 4 is devoted to the implementation issues, while in Section 5 two examples are discussed in detail: the first one is analytic and is aimed at comparing the conservativeness of the algorithm to the one of the well known tube based approach [24], while the second one is numeric and allows for a comparison of the different algorithm implementations. Finally, in Section 6 we draw some conclusions. For clarity of exposition, the proof of the main theoretical results is postponed to the Appendix.

Notation. The symbols $\succ$ and $\succeq$ (respectively $\prec$, and $\preceq$ ) are used to denote positive definite and semi-positive definite (respectively negative definite and semi-negative definite) matrices. The point-to-set distance from $\zeta$ to $\mathcal{Z}$ is $\operatorname{dist}(\zeta, \mathcal{Z}):=\inf \{\|\zeta-z\|, z \in \mathcal{Z}\}$.

\section{Problem statement}

\subsection{Stochastic system and probabilistic constraints}

Consider the following discrete-time linear system

$$
\left\{\begin{array}{l}
x_{t+1}=A x_{t}+B u_{t}+F w_{t} \quad t \geq 0 \\
y_{t}=C x_{t}+v_{t}
\end{array}\right.
$$

where $x_{t} \in \mathbb{R}^{n}$ is the state, $u_{t} \in \mathbb{R}^{m}$ is the input, $y_{t} \in$ $\mathbb{R}^{p}$ is the measured output and $w_{t} \in \mathbb{R}^{n_{w}}, v_{t} \in \mathbb{R}^{p}$ are two independent, zero-mean, white noise processes with covariance matrices $W \succeq 0$ and $V \succ 0$, respectively, and a-priori unbounded support. The pair $(A, C)$ is assumed to be observable, and the pairs $(A, B)$ and $(A, \tilde{F})$ are reachable, where matrix $\tilde{F}$ satisfies $\tilde{F} \tilde{F}^{T}=F W F^{T}$.

Polytopic constraints on the state and input variables of system (1) are imposed in a probabilistic way, i.e., it is required that, for all $t \geq 0$

$$
\begin{aligned}
& \mathbb{P}\left\{b_{r}^{T} x_{t} \geq x_{r}^{\max }\right\} \leq p_{r}^{x} \quad r=1, \ldots, n_{r} \\
& \mathbb{P}\left\{c_{s}^{T} u_{t} \geq u_{s}^{\max }\right\} \leq p_{s}^{u} \quad s=1, \ldots, n_{s}
\end{aligned}
$$

where $\mathbb{P}(\phi)$ denotes the probability of $\phi, b_{r}, c_{s}$ are constant vectors, $x_{r}^{\max }, u_{s}^{\max }$ are bounds for the state and control variables, and $p_{r}^{x}, p_{s}^{u}$ are design parameters. It is also assumed that the set of relations $b_{r}^{T} x \leq x_{r}^{\max }$, $r=1, \ldots, n_{r}$ (respectively, $c_{s}^{T} u \leq u_{s}^{\max }, s=1, \ldots, n_{s}$ ), defines a convex set $\mathbb{X}$ (respectively, $\mathbb{U})$ containing the origin in its interior.

\subsection{Regulator structure}

For system (1), we want to design a standard regulation scheme made by the state observer

$$
\hat{x}_{t+1}=A \hat{x}_{t}+B u_{t}+L_{t}\left(y_{t}-C \hat{x}_{t}\right)
$$

coupled with the feedback control law

$$
u_{t}=\bar{u}_{t}-K_{t}\left(\hat{x}_{t}-\bar{x}_{t}\right)
$$

where $\bar{x}$ is the state of the nominal model

$$
\bar{x}_{t+1}=A \bar{x}_{t}+B \bar{u}_{t}
$$

In (4), (5), the feedforward term $\bar{u}_{t}$ and the gains $L_{t}, K_{t}$ are design parameters to be selected to guarantee convergence properties and the fulfillment of the probabilistic constraints (2), (3). Letting

$$
\begin{aligned}
& e_{t}=x_{t}-\hat{x}_{t} \\
& \varepsilon_{t}=\hat{x}_{t}-\bar{x}_{t}
\end{aligned}
$$

from (7) one has

$$
\delta x_{t}=x_{t}-\bar{x}_{t}=e_{t}+\varepsilon_{t}
$$

Define also the vector $\sigma_{t}=\left[\begin{array}{cc}e_{t}^{T} & \varepsilon_{t}^{T}\end{array}\right]^{T}$ whose dynamics, according to (1)-(7), is described by

$$
\sigma_{t+1}=\Phi_{t} \sigma_{t}+\Psi_{t}\left[\begin{array}{l}
w_{t} \\
v_{t}
\end{array}\right]
$$

where

$\Phi_{t}=\left[\begin{array}{cc}A-L_{t} C & 0 \\ L_{t} C & A-B K_{t}\end{array}\right], \Psi_{t}=\left[\begin{array}{cc}F & -L_{t} \\ 0 & L_{t}\end{array}\right]$ 
In the following it is assumed that, by a proper initialization, i.e. $\mathbb{E}\left\{\sigma_{0}\right\}=0$, and recalling that the processes $v$ and $w$ are zero mean, the enlarged state $\sigma_{t}$ of system (9) is zero-mean, so that $\bar{x}_{t}=\mathbb{E}\left\{x_{t}\right\}$. Then, denoting by $\Sigma_{t}=\mathbb{E}\left\{\sigma_{t} \sigma_{t}^{T}\right\}$ and by $\Omega=\operatorname{diag}(W, V)$ the covariance matrices of $\sigma_{t}$ and $\left[w_{t}^{T} v_{t}^{T}\right]^{T}$ respectively, the evolution of $\Sigma_{t}$ is governed by

$$
\Sigma_{t+1}=\Phi_{t} \Sigma_{t} \Phi_{t}^{T}+\Psi_{t} \Omega \Psi_{t}^{T}
$$

By definition, also the variable $\delta x_{t}$ defined by (8) is zero mean and its covariance matrix $X_{t}$ can be derived from $\Sigma_{t}$ as $X_{t}=\mathbb{E}\left\{\delta x_{t} \delta x_{t}^{T}\right\}=\left[\begin{array}{ll}I & I\end{array}\right] \Sigma_{t}\left[\begin{array}{ll}I & I\end{array}\right]^{T}$. Finally, letting $\delta u_{t}=u_{t}-\bar{u}_{t}=-K_{t}\left(\hat{x}_{t}-\bar{x}_{t}\right)$, one has $\mathbb{E}\left\{\delta u_{t}\right\}=0$ and also the covariance matrix $U_{t}=\mathbb{E}\left\{\delta u_{t} \delta u_{t}^{T}\right\}$ can be obtained from $\Sigma_{t}$ as $U_{t}=\mathbb{E}\left\{K_{t} \varepsilon_{t} \varepsilon_{t}^{T} K_{t}^{T}\right\}=\left[\begin{array}{ll}0 & K_{t}\end{array}\right] \Sigma_{t}\left[\begin{array}{ll}0 & K_{t}\end{array}\right]^{T}$.

\subsection{Reformulation of the probabilistic constraints}

To incorporate the probabilistic constraints (2) and (3) into the MPC problem, we rely on the probabilistic approximation method and we reformulate them as deterministic ones (involving the stochastic variable secondorder description) at the price of suitable tightening. This, save for the case of Gaussian disturbances, induces some conservativeness.

Consider, in general, a random variable $z$ with mean value $\bar{z}=\mathbb{E}\{z\}$, variance $Z=\mathbb{E}\left\{(z-\bar{z})(z-\bar{z})^{T}\right\}$, and the probabilistic constraint

$$
\mathbb{P}\left\{h^{T} z \geq z^{\max }\right\} \leq p
$$

The following result, based on the Chebyshev - Cantelli inequality [22], has been proven in [21].

Proposition 1 Letting $f(p)=\sqrt{(1-p) / p}$, constraint (11) is verified if

$$
h^{T} \bar{z} \leq z^{\max }-\sqrt{h^{T} Z h} f(p)
$$

Note that this result can be proved without introducing any specific assumption on the distribution of $z$. If, on the other hand, $z$ can be assumed to be normally distributed, less conservative constraints can be obtained, as stated in the following result.

Proposition 2 Assume that $z$ is normally distributed. Then, constraint (11) is verified if (12) holds with $f(p)=$ $\mathcal{N}^{-1}(1-p)$ where $\mathcal{N}$ is the cumulative probability function of a Gaussian variable with zero mean and unitary variance.

In Propositions 1 and 2, the function $f(p)$ represents the level of constraint tightening on the mean value of $z$ needed to meet the probabilistic constraint (11). In case of unknown distribution (Proposition 1) the values of $f(p)$ are greater than in the Gaussian case (e.g., about an order of magnitude in the range $(0.1,0.4))$.

In view of Propositions 1 and 2, the probabilistic constraints (2)-(3), at time $i$, are verified provided that the following (deterministic) inequalities are satisfied.

$$
\begin{aligned}
& b_{r}^{T} \bar{x}_{i} \leq x_{r}^{\max }-\sqrt{b_{r}^{T} X_{i} b_{r}} f\left(p_{r}^{x}\right) \\
& c_{s}^{T} \bar{u}_{i} \leq u_{s}^{\max }-\sqrt{c_{s}^{T} U_{i} c_{s}} f\left(p_{s}^{u}\right)
\end{aligned}
$$

If the support of the noise terms $w_{k}$ and $v_{k}$ is unbounded, the definition of the state and control constraints in probabilistic terms is the only way to state feasible control problems. In case of bounded noise the comparison, in terms of conservativeness, between the probabilistic framework and the deterministic one is discussed in the example of Section 5.1.

\section{MPC algorithm: formulation and properties}

To formally state the MPC algorithm for the computation of the regulator parameters $\bar{u}_{t}, L_{t}, K_{t}$, the following notation will be adopted: given a variable $z$ or a matrix $Z$, at any time step $t$ we will denote by $z_{t+k}$ and $Z_{t+k}$, $k \geq 0$, their generic values in the future, while $z_{t+k \mid t}$ and $Z_{t+k \mid t}$ will represent their specific values computed based on the knowledge (e.g., measurements) available at time $t$. The main ingredients of the optimization problem are now introduced.

\subsection{Cost function}

Assume to be at time $t$ and denote by $\bar{u}_{t, \ldots, t+N-1}=$ $\left\{\bar{u}_{t}, \ldots, \bar{u}_{t+N-1}\right\}$ the nominal input sequence over a future prediction horizon of length $N$. Moreover, define by $K_{t, \ldots, t+N-1}=\left\{K_{t}, \ldots, K_{t+N-1}\right\}$, $L_{t, \ldots, t+N-1}=\left\{L_{t}, \ldots, L_{t+N-1}\right\}$ the sequences of the future control and observer, respectively, gains, and recall that the covariance $\Sigma_{t+k}=\mathbb{E}\left\{\sigma_{t+k} \sigma_{t+k}^{T}\right\}$ evolves, starting from $\Sigma_{t}$, according to (10).

The cost function to be minimized is the sum of two components, the first one $\left(J_{m}\right)$ accounts for the expected values of the future nominal inputs and states, while the second one $\left(J_{v}\right)$ is related to the variances of the future errors $e, \varepsilon$, and of the future inputs. Specifically, the overall performance index is

$J=J_{m}\left(\bar{x}_{t}, \bar{u}_{t, \ldots, t+N-1}\right)+J_{v}\left(\Sigma_{t}, K_{t, \ldots, t+N-1}, L_{t, \ldots, t+N-1}\right)$ 
where

$$
\begin{aligned}
& J_{m}=\sum_{i=t}^{t+N-1}\left\|\bar{x}_{i}\right\|_{Q}^{2}+\left\|\bar{u}_{i}\right\|_{R}^{2}+\left\|\bar{x}_{t+N}\right\|_{S}^{2} \\
& J_{v}=\mathbb{E}\left\{\sum_{i=t}^{t+N-1}\left\|x_{i}-\hat{x}_{i}\right\|_{Q_{L}}^{2}+\left\|x_{t+N}-\hat{x}_{t+N}\right\|_{S_{L}}^{2}\right\}+ \\
& \mathbb{E}\left\{\sum_{i=t}^{t+N-1}\left\|\hat{x}_{i}-\bar{x}_{i}\right\|_{Q}^{2}+\left\|u_{i}-\bar{u}_{i}\right\|_{R}^{2}+\left\|\hat{x}_{t+N}-\bar{x}_{t+N}\right\|_{S}^{2}\right\}
\end{aligned}
$$

where the positive definite and symmetric weights $Q$, $Q_{L}, S$, and $S_{L}$ must satisfy the following inequality

$$
\bar{Q}_{T}-S_{T}+\Phi^{T} S_{T} \Phi \preceq 0
$$

where $\Phi=\left[\begin{array}{cc}A-\bar{L} C & 0 \\ \bar{L} C & A-B \bar{K}\end{array}\right], \bar{Q}_{T}=\operatorname{diag}\left(Q_{L}, Q+\right.$ $\left.\bar{K}^{T} R \bar{K}\right), S_{T}=\operatorname{diag}\left(S_{L}, S\right)$, and $\bar{K}, \bar{L}$ must be chosen to guarantee that $\Phi$ is asymptotically stable.

By means of standard computations, it is possible to write the cost (16) as follows

$$
J_{v}=\sum_{i=t}^{t+N-1} \operatorname{tr}\left(Q_{T, i} \Sigma_{i}\right)+\operatorname{tr}\left(S_{T} \Sigma_{t+N}\right)
$$

where $Q_{T, i}=\operatorname{diag}\left(Q_{L}, Q+\bar{K}_{i}^{T} R \bar{K}_{i}\right)$. From (14)-(16), it is apparent that the goal is twofold: to drive the mean $\bar{x}$ to zero by acting on the nominal input component $\bar{u}_{t, \ldots, t+N-1}$ and to minimize the variance of $\sigma$ by acting on the gains $K_{t, \ldots, t+N-1}$ and $L_{t, \ldots, t+N-1}$. In addition, also the pair $\left(\bar{x}_{t}, \Sigma_{t}\right)$ must be considered as an additional argument of the MPC optimization, as later discussed, to guarantee recursive feasibility.

\subsection{Terminal constraints}

As usual in stabilizing MPC, see e.g. [23], some terminal constraints must be considered. In our setup, the mean $\bar{x}_{t+N}$ and the variance $\Sigma_{t+N}$ at the end of the prediction horizon must satisfy

$$
\begin{aligned}
& \bar{x}_{t+N} \in \overline{\mathbb{X}}_{F} \\
& \Sigma_{t+N} \preceq \bar{\Sigma}
\end{aligned}
$$

where $\overline{\mathbb{X}}_{F}$ is a positively invariant set such that

$$
(A-B \bar{K}) \bar{x} \in \overline{\mathbb{X}}_{F} \quad \forall \bar{x} \in \overline{\mathbb{X}}_{F}
$$

while $\bar{\Sigma}$ is the steady-state solution of the Lyapunov equation (10), i.e.,

$$
\bar{\Sigma}=\Phi \bar{\Sigma} \Phi^{T}+\Psi \bar{\Omega} \Psi^{T}
$$

where $\Psi=\left[\begin{array}{cc}F & -\bar{L} \\ 0 & \bar{L}\end{array}\right]$ and $\bar{\Omega}=\operatorname{diag}(\bar{W}, \bar{V})$ is built by considering (arbitrary) noise variances $\bar{W} \succeq W$ and $\bar{V} \succeq$ $V$. In addition, and consistently with (13), the following coupling conditions must be verified.

$$
\begin{array}{r}
b_{r}^{T} \bar{x} \leq x_{r}^{\max }-\sqrt{b_{r}^{T} \bar{X} b_{r}} f\left(p_{r}^{x}\right), r=1, \ldots, n_{r} \\
-c_{s}^{T} \bar{K} \bar{x} \leq u_{s}^{\max }-\sqrt{c_{s}^{T} \bar{U} c_{s}} f\left(p_{s}^{u}\right), s=1, \ldots, n_{s}
\end{array}
$$

for all $\bar{x} \in \overline{\mathbb{X}}_{F}$, where

$$
\bar{X}=\left[\begin{array}{ll}
I & I
\end{array}\right] \bar{\Sigma}\left[\begin{array}{ll}
I & I
\end{array}\right]^{T}, \bar{U}=\left[\begin{array}{ll}
0 & \bar{K}
\end{array}\right] \bar{\Sigma}\left[\begin{array}{ll}
0 & \bar{K}
\end{array}\right]^{T}
$$

As it will be shown (see Theorem 1), (19) and (20) allow for recursive feasibility of the control scheme and enforce mean square convergence properties.

In (22), the choice of $\bar{\Omega}$ is subject to a tradeoff. In fact, large variances $\bar{W}$ and $\bar{V}$ result in large $\bar{\Sigma}$ and, in view of (24), large $\bar{X}$ and $\bar{U}$. This enlarges the terminal constraint (20) but, on the other hand, reduces the size of the terminal set $\mathbb{X}_{F}$ compatible with (23).

\subsection{Statement of the stochastic $M P C(S-M P C)$ problem}

The formulation of the main S-MPC problem requires a preliminary discussion concerning the initialization. In principle, and in order to use the most recent information available on the state, at each time instant it would be natural to set the current value of the nominal state $\bar{x}_{t \mid t}$ to $\hat{x}_{t}$ and the covariance $\Sigma_{t \mid t}$ to $\operatorname{diag}\left(\Sigma_{11, t \mid t-1}, 0\right)$, where $\Sigma_{11, t \mid t-1}$ is the covariance of state prediction error $e$ obtained using the observer (4). However, since we do not exclude the possibility of unbounded disturbances, in some cases this choice could lead to infeasible optimization problems. On the other hand, and in view of the terminal constraints (19), (20), it is quite easy to see that recursive feasibility is guaranteed provided that $\bar{x}$ is updated according to the prediction equation (6), which corresponds to the variance update given by (10). These considerations motivate the choice of accounting for the initial conditions $\left(\bar{x}_{t}, \Sigma_{t}\right)$ as free variables, which will be selected by the control algorithm (based on feasibility and optimality of the MPC optimization problem defined below) according to the following alternative strategies:S1) reset of the initial state: $\bar{x}_{t \mid t}=\hat{x}_{t}$, $\Sigma_{t \mid t}=\operatorname{diag}\left(\Sigma_{11, t \mid t-1}, 0\right)$; S2) prediction: $\bar{x}_{t \mid t}=\bar{x}_{t \mid t-1}$, $\Sigma_{t \mid t}=\Sigma_{t \mid t-1}$. The S-MPC problem can now be stated.

S-MPC problem: at any time instant $t$ solve

$$
\min _{\bar{x}_{t}, \Sigma_{t}, \bar{u}_{t, \ldots, t+N-1}, K_{t, \ldots, t+N-1}, L_{t, \ldots, t+N-1}} J
$$

where $J$ is defined in (14), (15), (16), subject to 
- the dynamics (6) and (10);

- the constraints (13) for all $i=t, \ldots, t+N-1, r=$ $1, \ldots, n_{r}, s=1, \ldots, n_{s}$;

- the initialization constraint (corresponding to the choice between strategies S1 and S2)

$$
\left(\bar{x}_{t}, \Sigma_{t}\right) \in\left\{\left(\hat{x}_{t}, \operatorname{diag}\left(\Sigma_{11, t \mid t-1}, 0\right)\right),\left(\bar{x}_{t \mid t-1}, \Sigma_{t \mid t-1}\right)\right\}
$$

- the terminal constraints (19), (20).

Denoting by $\bar{u}_{t, \ldots, t+N-1 \mid t}=\left\{\bar{u}_{t \mid t}, \ldots, \bar{u}_{t+N-1 \mid t}\right\}$, $K_{t, \ldots, t+N-1 \mid t}=\left\{K_{t \mid t}, \ldots\right.$, $\left.K_{t+N-1 \mid t}\right\}, L_{t, \ldots, t+N-1 \mid t}=\left\{L_{t \mid t}, \ldots, L_{t+N-1 \mid t}\right\}$, and $\left(\bar{x}_{t \mid t}, \Sigma_{t \mid t}\right)$ the optimal solution to the S-MPC problem, the feedback control law actually used is then given by (5) with $\bar{u}_{t}=\bar{u}_{t \mid t}, K_{t}=K_{t \mid t}$, and the state observation evolves as in (4) with $L_{t}=L_{t \mid t}$.

We define the S-MPC problem feasibility set as $\Xi:=\left\{\left(\bar{x}_{0}, \Sigma_{0}\right): \exists \bar{u}_{0, \ldots, N-1}, K_{0, \ldots, N-1}, L_{0, \ldots, N-1}\right.$ such that (6), (10), and (13) hold for all $k=0, \ldots, N-1$ and (19), (20) are verified $\}$

Some comments are in order.

1) At the initial time $t=0$, the algorithm must be initialized by setting $\bar{x}_{0 \mid 0}=\hat{x}_{0}$ and $\Sigma_{0 \mid 0}=\operatorname{diag}\left(\Sigma_{11,0}, 0\right)$. In view of this, feasibility at time $t=0$ amounts to $\left(\hat{x}_{0}, \Sigma_{0 \mid 0}\right) \in \Xi$.

2) The binary choice between strategies S1 and S2 requires to solve at any time instant two optimization problems. However, the following sequential procedure can be adopted to reduce the average overall computational burden: the optimization problem corresponding to strategy S1 is first solved and, if it is infeasible, strategy S2 must be solved and adopted. On the contrary, if the problem with strategy $\mathrm{S} 1$ is feasible, it is possible to compare the resulting value of the optimal cost function with the value of the cost using the sequences $\left\{\bar{u}_{t \mid t-1}, \ldots, \bar{u}_{t+N-2 \mid t-1},-\bar{K} \bar{x}_{t+N-1 \mid t}\right\}$, $\left\{K_{t \mid t-1}, \ldots, K_{t+N-2 \mid t-1}, \bar{K}\right\},\left\{L_{t \mid t-1}, \ldots, L_{t+N-2 \mid t-1}, \bar{L}\right\}$. If the optimal cost with strategy $\mathrm{S} 1$ is lower, strategy $\mathrm{S} 1$ can be used without solving the MPC problem for strategy S2. This does not guarantee optimality, but the convergence properties of the method stated in the result below are recovered and the computational effort is reduced.

Now we are in the position to state the main result concerning the convergence properties of the algorithm.

Theorem 1 If, at $t=0$, the $S$-MPC problem admits a solution, it is recursively feasible and the state and the input probabilistic constraints (2) and (3) are satisfied for all $t \geq 0$. Furthermore, if there exists $\rho \in(0,1)$ such that the noise variance $\Omega$ verifies

$$
\frac{\left(N+\frac{\beta}{\alpha}\right)}{\alpha} \operatorname{tr}\left(S_{T} \Psi \Omega \Psi^{T}\right)<\min \left(\rho \bar{\sigma}^{2}, \rho \lambda_{\min }(\bar{\Sigma})\right)
$$

where $\bar{\sigma}$ is the maximum radius of a ball, centered at the origin, included in $\overline{\mathbb{X}}_{F}$, and

$$
\begin{aligned}
& \alpha=\min \left\{\lambda_{\min }(Q), \operatorname{tr}\left\{Q^{-1}+Q_{L}^{-1}\right\}^{-1}\right\} \\
& \beta=\max \left\{\lambda_{\max }(S), \operatorname{tr}\left\{S_{T}\right\}\right\}
\end{aligned}
$$

then, as $t \rightarrow+\infty$

$$
\operatorname{dist}\left(\left\|\bar{x}_{t}\right\|^{2}+\operatorname{tr}\left\{\Sigma_{t \mid t}\right\},\left[0, \frac{1}{\alpha}\left(N+\frac{\beta}{\alpha}\right) \operatorname{tr}\left(S_{T} \Psi \Omega \Psi^{T}\right)\right]\right) \rightarrow 0
$$

Note that, as expected, for smaller and smaller values of $\Omega$, also $\left\|\bar{x}_{t}\right\|$ and $\operatorname{tr}\left\{\Sigma_{t \mid t}\right\}$ tend to zero.

\section{Implementation issues}

In this section two issues are addressed. First, the non linear constraints (13) and the non linear dependence of the covariance evolution, see (10), on $K_{t, \ldots, t+N-1}, L_{t, \ldots, t+N-1}$ make the numerical solution of S-MPC impractical. In sections 4.1 and 4.2 two possible solutions are described, allowing to cast the S-MPC problem as a quadratic one, with linear constraints. The second issue concerns the fact that, in our framework, deterministic constraints on input variable (e.g., saturations) are not accounted for. In Section 4.3 we propose some possible solutions to this problem.

\subsection{Approximation of S-MPC for allowing a solution with LMIs}

A solution, based on an approximation of S-MPC characterized by linear constraints solely, is now presented. First define $A^{D}=\sqrt{2} A, B^{D}=\sqrt{2} B, C^{D}=\sqrt{2} C$, and $V^{D}=2 V$ and let the auxiliary gain matrices $\bar{K}$ and $\bar{L}$ be selected according to the following assumption.

Assumption 1 The gains $\bar{K}$ and $\bar{L}$ are computed as the steady-state gains of the $L Q G$ regulator for the system $\left(A^{D}, B^{D}, C^{D}\right)$, with state and control weights $Q$ and $R$, and noise covariances $\bar{W} \succeq W$ and $\bar{V} \succeq V^{D}$.

Note that, if a gain matrix $\bar{K}$ (respectively $\bar{L}$ ) is stabilizing for $\left(A^{D}-B^{D} \bar{K}\right)=\sqrt{2}(A-B \bar{K})$ (respectively $\left.\left(A^{D}-\bar{L} C^{D}\right)=\sqrt{2}(A-\bar{L} C)\right)$, it is also stabilizing for $(A-B \bar{K})($ respectively $(A-\bar{L} C))$, i.e., for the original system. The following preliminary result can be stated.

Lemma 1 Define $A_{L_{t}}^{D}=A^{D}-L_{t} C^{D}, A_{K_{t}}^{D}=A^{D}-$ $B^{D} K_{t}$, the block diagonal matrix $\Sigma_{t}^{D}=\operatorname{diag}\left(\Sigma_{11, t}^{D}, \Sigma_{22, t}^{D}\right)$, $\Sigma_{11, t}^{D} \in \mathbb{R}^{n \times n}, \Sigma_{22, t}^{D} \in \mathbb{R}^{n \times n}$ and the update equations

$$
\begin{aligned}
\Sigma_{11, t+1}^{D}= & A_{L_{t}}^{D} \Sigma_{11, t}^{D}\left(A_{L_{t}}^{D}\right)^{T}+F W F^{T}+L_{t} V^{D} L_{t}^{T} \\
\Sigma_{22, t+1}^{D}= & A_{K_{t}}^{D} \Sigma_{22, t}^{D}\left(A_{K_{t}}^{D}\right)^{T}+L_{t} C^{D} \Sigma_{11, t}^{D} C^{D} L_{t}^{T} \\
& +L_{t} V^{D} L_{t}^{T}
\end{aligned}
$$


Then

I) $\Sigma_{t}^{D} \succeq \Sigma_{t}$ implies that $\Sigma_{t+1}^{D}=\operatorname{diag}\left(\Sigma_{11, t+1}^{D}, \Sigma_{22, t+1}^{D}\right) \succeq$ $\Sigma_{t+1}$.

II) We can rewrite as LMIs the following inequalities

$$
\begin{aligned}
\Sigma_{11, t+1}^{D} \succeq A_{L_{t}}^{D} \Sigma_{11, t}^{D}\left(A_{L_{t}}^{D}\right)^{T}+F W F^{T}+L_{t} V^{D} L_{t}^{T} \\
\Sigma_{22, t+1}^{D} \succeq A_{K_{t}}^{D} \Sigma_{22, t}^{D}\left(A_{K_{t}}^{D}\right)^{T}+L_{t} C^{D} \Sigma_{11, t}^{D} C^{D} L_{t}^{T} \\
\\
+L_{t} V^{D} L_{t}^{T}
\end{aligned}
$$

Based on Lemma 1-II, we can reformulate the original problem so that the covariance matrix $\Sigma^{D}$ is used instead of $\Sigma$. Accordingly, the update equation (10) is replaced by (29) and the S-MPC problem is recast as an LMI one (see Appendix B).

As for the nonlinear dependence of the inequalities (13) on the covariance matrices $X_{t}$ and $U_{t}$, it is possible to prove that they are satisfied if

$$
\begin{aligned}
& b_{r}^{T} \bar{x}_{i} \leq\left(1-0.5 \alpha_{x}\right) x_{r}^{\text {max }}-\frac{b_{r}^{T} X_{i} b_{r}}{2 \alpha_{x} x_{r}^{\text {max }}} f\left(p_{r}^{x}\right)^{2} \\
& c_{s}^{T} \bar{u}_{i} \leq\left(1-0.5 \alpha_{u}\right) u_{s}^{\text {max }}-\frac{c_{s}^{T} U_{i} c_{s}}{2 \alpha_{u} u_{s}^{\text {max }}} f\left(p_{s}^{u}\right)^{2}
\end{aligned}
$$

where $\alpha_{x} \in(0,1]$ and $\alpha_{u} \in(0,1]$ are free design parameters. Also, note that $X_{t} \preceq\left[\begin{array}{ll}I & I\end{array}\right] \Sigma_{t}^{D}\left[\begin{array}{ll}I & I\end{array}\right]^{T}=$ $\Sigma_{11, t}^{D}+\Sigma_{22, t}^{D}$ and that $U_{t} \preceq\left[\begin{array}{ll}0 & K_{t}\end{array}\right] \Sigma_{t}^{D}\left[\begin{array}{ll}0 & K_{t}\end{array}\right]^{T}=$ $K_{t} \Sigma_{22, t}^{D} K_{t}^{T}$ so that, defining $X_{t}^{D}=\Sigma_{11, t}^{D}+\Sigma_{22, t}^{D}$ and $U_{t}^{D}=K_{t} \Sigma_{22, t}^{D} K_{t}^{T}$, (31) can be written as follows

$$
\begin{aligned}
& b_{r}^{T} \bar{x}_{i} \leq\left(1-0.5 \alpha_{x}\right) x_{r}^{\max }-\frac{b_{r}^{T} X_{i}^{D} b_{r}}{2 \alpha_{x} x_{r}^{\text {max }}} f\left(p_{r}^{x}\right)^{2} \\
& c_{s}^{T} \bar{u}_{i} \leq\left(1-0.5 \alpha_{u}\right) u_{s}^{\text {max }}-\frac{c_{s}^{T} U_{i}^{D} c_{s}}{2 \alpha_{u} u_{s}^{\text {max }}} f\left(p_{s}^{u}\right)^{2}
\end{aligned}
$$

Note that the reformulation of (13) into (32) has been performed at the price of additional constraint tightening. For example, on the right hand side of (32a), $x_{r}^{\max }$ is replaced by $\left(1-0.5 \alpha^{x}\right) x_{r}^{\max }$, which significantly reduces the size of the constraint set. Parameter $\alpha^{x}$ cannot be reduced at will, since it also appears at the denominator in the second additive term.

In view of Assumption 1 and resorting to the separation principle, it is possible to show [11] that the solution $\bar{\Sigma}^{D}$ to the steady-state equation

$$
\bar{\Sigma}^{D}=\Phi^{D} \bar{\Sigma}^{D}\left(\Phi^{D}\right)^{T}+\Psi \bar{\Omega} \Psi^{T}
$$

is block-diagonal, i.e., $\bar{\Sigma}^{D}=\operatorname{diag}\left(\bar{\Sigma}_{11}^{D}, \bar{\Sigma}_{22}^{D}\right)$, where $\Phi^{D}=\left[\begin{array}{cc}A^{D}-\bar{L} C^{D} & 0 \\ \bar{L} C^{D} & A^{D}-B^{D} \bar{K}\end{array}\right]$
The terminal constraint (20) must be transformed into $\Sigma_{t+N}^{D} \preceq \bar{\Sigma}^{D}$, which corresponds to setting

$$
\Sigma_{11, t+N}^{D} \preceq \bar{\Sigma}_{11}^{D}, \Sigma_{22, t+N}^{D} \preceq \bar{\Sigma}_{22}^{D}
$$

Defining $\bar{X}^{D}=\bar{\Sigma}_{11}^{D}+\bar{\Sigma}_{22}^{D}$ and $\bar{U}^{D}=\bar{K} \bar{\Sigma}_{22}^{D} \bar{K}^{T}$, the terminal set condition (23) must now be reformulated as

$$
\begin{array}{r}
b_{r}^{T} \bar{x} \leq\left(1-0.5 \alpha^{x}\right) x_{r}^{\max }-\frac{b_{r}^{T} \bar{X}^{D} b_{r}}{2 \alpha^{x} x_{r}^{\text {max }}} f\left(p_{r}^{x}\right)^{2} \\
-c_{s}^{T} \bar{K} \bar{x} \leq\left(1-0.5 \alpha^{u}\right) u_{s}^{\text {max }}-\frac{c_{s}^{T} \bar{U}^{D} c_{s}}{2 \alpha^{u} u_{s}^{\text {max }}} f\left(p_{s}^{u}\right)^{2}
\end{array}
$$

for all $\bar{x} \in \overline{\mathbb{X}}_{F}$.

Also $J_{v}$ must be reformulated. Indeed

$$
\begin{aligned}
J_{v} \leq J_{v}^{D} & =\sum_{i=t}^{t+N-1} \operatorname{tr}\left\{Q_{L} \Sigma_{11, i}^{D}+Q \Sigma_{22, i}^{D}+R K_{i} \Sigma_{22, i}^{D} K_{i}^{T}\right\} \\
& +\operatorname{tr}\left\{S_{L} \Sigma_{11, t+N}^{D}+S \Sigma_{22, t+N}^{D}\right\}
\end{aligned}
$$

where the terminal weights $S$ and $S_{L}$ must now satisfy the following Lyapunov-like inequalities

$$
\begin{aligned}
& \left(\bar{A}_{K}^{D}\right)^{T} S \bar{A}_{K}^{D}-S+Q+\bar{K}^{T} R \bar{K} \preceq 0 \\
& \left(\bar{A}_{L}^{D}\right)^{T} S_{L} \bar{A}_{L}^{D}-S_{L}+Q_{L}+\left(C^{D}\right)^{T} \bar{L}^{T} S \bar{L} C^{D} \preceq 0
\end{aligned}
$$

where $\bar{A}_{K}^{D}=A^{D}-B^{D} \bar{K}$ and $\bar{A}_{L}^{D}=A^{D}-\bar{L} C^{D}$. It is now possible to formally state the S-MPCl problem.

S-MPCl problem: at any time instant $t$ solve

$$
\min _{\bar{x}_{t}, \Sigma_{11, t}^{D}, \Sigma_{22, t}^{D}, \bar{u}_{t, \ldots, t+N-1}, K_{t, \ldots, t+N-1}, L_{t, \ldots, t+N-1}} J
$$

where $J$ is defined in (14), (15), (36), subject to

- the dynamics (6) and (29);

- the linear constraints (32) for all $i=t, \ldots, t+N-1$, $r=1, \ldots, n_{r}, s=1, \ldots, n_{s}$;

- the initialization constraint, corresponding to the choice between strategies S1 and S2, i.e., $\left(\bar{x}_{t}, \Sigma_{11, t}^{D}, \Sigma_{22, t}^{D}\right) \in\left\{\left(\hat{x}_{t}, \Sigma_{11, t \mid t-1}^{D}, 0\right),\left(\bar{x}_{t \mid t-1}, \Sigma_{11, t \mid t-1}^{D}, \Sigma_{22, t \mid t-1}^{D}\right)\right\}$

- the terminal constraints (19), (34).

The following corollary follows from Theorem 1.

Corollary $1 \mathrm{If}$, at time $t=0$, the $S$-MPCl problem admits a solution, it is recursively feasible and the state and input probabilistic constraints (2) and (3) are satisfied for all $t \geq 0$. Furthermore, if there exists $\rho \in(0,1)$ such that the noise variance $\Omega^{D}=\operatorname{diag}\left(W, V^{D}\right)$ verifies

$$
\frac{\left(N+\frac{\beta}{\alpha}\right)}{\alpha} \operatorname{tr}\left(S_{T} \Psi \Omega^{D} \Psi^{T}\right)<\min \left(\rho \bar{\sigma}^{2}, \rho \lambda_{\min }\left(\bar{\Sigma}^{D}\right)\right)
$$


then, as $t \rightarrow+\infty$

$\operatorname{dist}\left(\left\|\bar{x}_{t}\right\|^{2}+\operatorname{tr}\left\{\Sigma_{t \mid t}^{D}\right\},\left[0, \frac{1}{\alpha}\left(N+\frac{\beta}{\alpha}\right) \operatorname{tr}\left(S_{T} \Psi \Omega^{D} \Psi^{T}\right)\right]\right) \rightarrow 0$

The proof of Corollary 1 readily follows from the proof of Theorem 1. For details please see [10].

\subsection{Approximation of $S-M P C$ with constant gains}

The solution presented in the following is characterized by a great simplicity and consists in setting $L_{t}=\bar{L}$ and $K_{t}=\bar{K}$ for all $t \geq 0$. In this case, the value of $\Sigma_{t+k}$ (and therefore of $X_{t+k}$ and $U_{t+k}$ ) can be directly computed for any $k>0$ by means of (10) as soon as $\Sigma_{t}$ is given. As a byproduct, the nonlinearity in the constraints (13) does not carry about implementation problems. Therefore, this solution has a twofold advantage: first, it is simple and requires an extremely lightweight implementation; secondly, it allows for the use of nonlinear less conservative constraint formulations. In this simplified framework, the following S-MPCc problem can be stated.

S-MPCc problem: at any time instant $t$ solve

$$
\min _{\bar{x}_{t}, \Sigma_{t}, \bar{u}_{t, \ldots, t+N-1}} J
$$

where $J$ is defined in (14), (15), (16), subject to

- the dynamics (6), with $K_{t}=\bar{K}$, and

$$
\Sigma_{t+1}=\Phi \Sigma_{t} \Phi^{T}+\Psi \Omega \Psi^{T}
$$

- the constraints (13) for all $i=t, \ldots, t+N-1, r=$ $1, \ldots, n_{r}, s=1, \ldots, n_{s}$;

- the initialization constraint (25);

- the terminal constraints (19), (20).

An additional remark is due. The term $J_{v}$ in (18) does not depend only on the control and observer gain sequences $K_{t, \ldots, t+N-1}, L_{t, \ldots, t+N-1}$, but also on the initial condition $\Sigma_{t}$. Therefore, it is not possible to discard it in this simplified formulation.

The following corollary can be derived from Theorem 1.

Corollary 2 If, at time $t=0$, the $S$-MPCc problem admits a solution, it is recursively feasible and the state and input probabilistic constraints (2) and (3) are satisfied for all $t \geq 0$. Furthermore, if there exists $\rho \in(0,1)$ such that the noise variance $\Omega$ verifies (26), then, ast $\rightarrow+\infty$, (28) holds.

The proof of Corollary 2 readily follows from the proof of Theorem 1. For details please see [10].

\subsection{Boundedness of the input variables}

The S-MPC scheme described in the previous sections cannot handle hard constraints on the input variables. However, in general input variables are bounded in practice, and may be subject to

$$
H u_{t} \leq \mathbf{1}
$$

where $H \in \mathbb{R}^{n_{H} \times m}$ is a design matrix and $\mathbf{1}$ is a vector of dimension $n_{H}$ whose entries are equal to 1 . Three possible approaches are proposed to account for this case. 1) Inequalities (40) can be stated as additive probabilistic constraints (3) with small violation probabilities $p_{s}^{u}$. This solution, although not guaranteeing satisfaction of (40) with probability 1 , is simple and easy.

2 ) In the S-MPCc scheme, define the gain matrix $\bar{K}$ in such a way that $A-B \bar{K}$ is asymptotically stable and, at the same time, $H \bar{K}=0$. From (5), it follows that $H u_{t}=H \bar{u}_{t}+H \bar{K}\left(\hat{x}_{t}-\bar{x}_{t}\right)=H \bar{u}_{t}$. Therefore, to verify (40) it is sufficient to include in the problem formulation the deterministic constraint $H \bar{u}_{t} \leq \mathbf{1}$.

$3)$ In the S-MPCc scheme, if probabilistic constraints on $u$ are absent, replace (5) with $u_{t}=\bar{u}_{t}$ and set $H \bar{u}_{t} \leq \mathbf{1}$ in the S-MPC optimization problem to verify (40). If we also define $\hat{u}_{t}=\bar{u}_{t}-\bar{K}\left(\hat{x}_{t}-\bar{x}_{t}\right)$ as the input to equation (4), the dynamics of variable $\sigma_{t}$ is given by (9) with

$\Phi_{t}=\left[\begin{array}{cc}A-\bar{L} C & B \bar{K} \\ \bar{L} C & A-B \bar{K}\end{array}\right]$

and the arguments follow similarly to those proposed in the paper. It is worth mentioning, however, that matrix $\Phi_{t}$ must be asymptotically stable, which requires asymptotic stability of $A$.

\section{Examples}

In this section a comparison between the characteristics of the proposed method and robust tube-based MPC $[25,24]$ is first discussed. Then, the performances of the algorithms S-MPCl and S-MPCc described in Section 4 are discussed with reference to a numerical example.

\subsection{Simple analytic example: comparison between the probabilistic and the deterministic robust MPC}

Consider the scalar system $x_{t+1}=a x_{t}+u_{t}+w_{t}$, where $0<a<1, w \in\left[-w_{\max }, w_{\max }\right], w_{\max }>0$, and the measurable state is constrained as follows

$$
x_{t} \leq x_{\max }
$$

The limitations imposed by the deterministic robust MPC algorithm developed in [25] and by the probabilistic (state-feedback) method described in this paper are now compared. For both the algorithms, the control law $u_{t}=\bar{u}_{t}$ is considered, where $\bar{u}$ is the input of the 


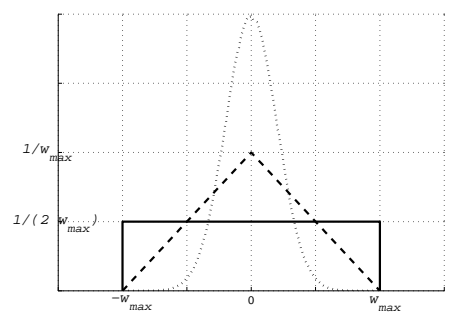

Fig. 1. Distributions: uniform (case A, solid line), triangular (case B, dashed line), truncated Gaussian (case C, dotted line).

nominal/average system $\bar{x}_{t+1}=a \bar{x}_{t}+b \bar{u}_{t}$. This control law is equivalent to (5), where it has been set $K_{t}=0$. In the probabilistic approach, we allow the constraint (41) to be violated with probability $p$, i.e.,

$$
\mathbb{P}\left\{x \geq x_{\max }\right\} \leq p
$$

To verify (41) and (42) the tightened constraint $\bar{x}_{k} \leq$ $x_{\max }-\Delta x$ must be fulfilled in both the approaches where, in case of [24], $\Delta x=\Delta x_{R P I}=\sum_{i=0}^{+\infty} a^{i} w_{\max }=$ $\frac{1}{1-a} w_{\max }$ while, having defined $w$ as a stochastic process with zero mean and variance $W$, in the probabilistic framework $\Delta x=\Delta x_{S}(p)=\sqrt{X(1-p) / p}$, and $X$ is the steady state variance satisfying the algebraic equation $X=a^{2} X+W$, i.e. $X=W /\left(1-a^{2}\right)$. Notably, $W$ takes different values depending upon the noise distribution. It results that the deterministic tightened constraints are more conservative provided that $\Delta x_{S}(p)<\Delta x_{R P I}$, i.e.

$$
p>\frac{(1-a)^{2}}{b\left(1-a^{2}\right)+(1-a)^{2}}
$$

Consider now the three distributions depicted in Figure 1 with $W=w_{\max }^{2} / b$, with A) $b=3$ for uniform distribution; B) $b=18$ for triangular distribution; C) $b=25$ for truncated Gaussian distribution.

Setting, for example, $a=0.9$, condition (43) is verified for $p>0.0172$ in case A), $p>0.0029$ in case B), and $p>0.0021$ in case $\mathrm{C}$ ). Note that, although formally truncated, the distribution in case C) can be well approximated with a non-truncated Gaussian distribution: if this information were available, one could use $\Delta x_{S}(p)=\sqrt{X} \mathcal{N}^{-1}(1-p)$ for constraint tightening, and in this case $\Delta x_{S}(p)<\Delta x_{R P I}$ would be verified with $p>1-\mathcal{N}\left(\frac{\left(1-a^{2}\right) b}{(1-a)^{2}}\right) \simeq 0$.

\subsection{Simulation example}

The example shown in this section is inspired by [25].

We take $A=\left[\begin{array}{ll}1 & 1 \\ 0 & 1\end{array}\right], B=\left[\begin{array}{c}0.5 \\ 1\end{array}\right], F=I_{2}, C=I_{2}$ and we assume that noise is Gaussian, with $W=0.01 I_{2}$ and

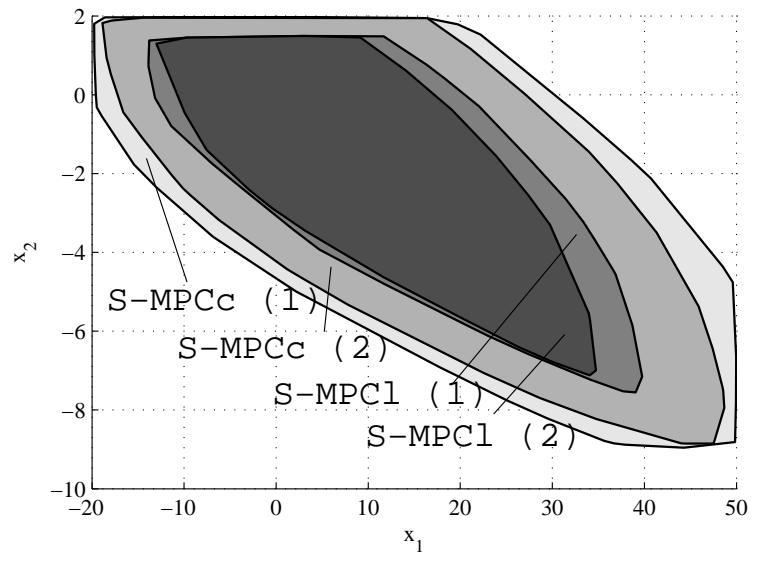

Fig. 2. Plots of the feasibility sets for S-MPCc (1), S-MPCc (2), S-MPCl (1), S-MPCl (2)

$V=10^{-4} I_{2}$. The probabilistic constraints are $\mathbb{P}\left\{x_{2} \geq\right.$ $2\} \leq 0.1, \mathbb{P}\{u \geq 1\} \leq 0.1$, and $\mathbb{P}\{-u \geq 1\} \leq 0.1$. In (14), (15), and (18) we set $Q_{L}=Q=I_{2}, R=0.01$, and $N=9$.

In Figure 2 we compare the feasible sets obtained with the methods presented in Section 4, with different assumptions concerning the noise (namely S-MPCc (1), SMPCc (2), S-MPCl (1), S-MPCl (2), where (1) denotes the case of Gaussian distribution and (2) denotes the case when the distribution is unknown). Apparently, in view of the linearization of the constraints (see the discussion after (32)), the S-MPCl algorithm is more conservative than S-MPCc. On the other hand, concerning the dimension of the obtained feasibility set, in this case the use of the Chebyshev - Cantelli inequality does not carry about a dramatic performance degradation in terms of conservativeness.

A 200-runs Montecarlo simulation campaign has been carried out for testing the probabilistic properties of the algorithms, with initial conditions $(5,-1.5)$. The fact that the control and estimation gains are free variables makes the transient behaviour of the state responses in case of $\mathrm{S}-\mathrm{MPCl}$ more performing and reduces the variance of the dynamic state response (at the price of a more reactive input response), with respect to the case when S-MPCc is used. For example, the maximum variance of $x_{1}(k)$ (resp. of $x_{1}(k)$ ) is about 0.33 (resp. 0.036) in case of S-MPCc (1) and (2), while it results about 0.25 (resp. 0.035) in case of S-MPCl (1) and (2). On the other hand, the maximum variance of $u(k)$ is about 0.006 in case of S-MPCc, while it is 0.008 in case of S-MPCl.

\section{Conclusions}

The main features of the proposed probabilistic MPC algorithm lie in its simplicity and in its light-weight computational load, both in the off-line design phase and in the online implementation. This allows for the application of the S-MPC scheme to medium/large-scale prob- 
lems, for systems affected by general disturbances.

The main limitations with respect to existing approaches are due to the difficulty to cope with saturations in the input variables and in the suboptimality of the solution when the Cantelli inequality is used instead of the full noise characterization. Future work will address these issues. Also, future developments will focus on the use of the proposed scheme in challenging application problems, such as the control of micro-grids in presence of renewable stochastic energy sources. The application of the algorithm to cope with linear time-varying systems is envisaged, while its extension to distributed implementations is currently underway.

\section{Acknowledgements}

We are indebted to B. Picasso for fruitful discussions.

\section{A Proof of the main Theorem 1}

Recursive feasibility is first proved. Assume that, at time instant $t$, a feasible solution of S-MPC is available, i.e., $\left(\bar{x}_{t \mid t}, \Sigma_{t \mid t}\right) \in \Xi$ with optimal sequences $\bar{u}_{t, \ldots, t+N-1 \mid t}, K_{t, \ldots, t+N-1 \mid t}$, and $L_{t, \ldots, t+N-1 \mid t}$. We prove that at time $t+1$ a feasible solution exists, i.e., in view of the initialization strategy S2, $\left(\bar{x}_{t+1 \mid t}, \Sigma_{t+1 \mid t}\right) \in \Xi$ with admissible sequences $\bar{u}_{t+1, \ldots, t+N \mid t}^{f}=\left\{\bar{u}_{t+1 \mid t}, \ldots, \bar{u}_{t+N-1 \mid t},-\bar{K} \bar{x}_{t+N \mid t}\right\}$, $K_{t+1, \ldots, t+N \mid t}^{f}=\left\{K_{t+1 \mid t}, \ldots, K_{t+N-1 \mid t}, \bar{K}\right\}$, and $L_{t+1, \ldots, t+N \mid t}^{f}=\left\{L_{t+1 \mid t}, \ldots, L_{t+N-1 \mid t}, \bar{L}\right\}$.

Constraint (13a) is verified for all pairs $\left(\bar{x}_{t+1+k \mid t}, X_{t+1+k \mid t}\right)$, $k=0, \ldots, N-2$, in view of the feasibility of S-MPC at time $t$. Furthermore, in view of (19), (20), (24), and the condition (23a), we have that $b^{T} \bar{x}_{t+N \mid t} \leq x^{\max }-\sqrt{b^{T} X_{t+N \mid t} b} f\left(p_{r}^{x}\right)$, i.e., constraint (13a) is verified.

Analogously, constraint (13b) is verified for all pairs $\left(\bar{u}_{t+1+k \mid t}, U_{t+1+k \mid t}\right), k=0, \ldots, N-2$, in view of the feasibility of S-MPC at time $t$. Furthermore, in view of (19), (20), (24), and the condition (23b), we have that $-c^{T} \bar{K} \bar{x}_{t+N \mid t} \leq u^{\max }-\sqrt{c^{T} U_{t+N \mid t} c} f\left(p_{s}^{u}\right)$, i.e., constraint (13b) is verified.

In view of (19) and of the invariance property (21) it follows that $\bar{x}_{t+N+1 \mid t}=(A-B \bar{K}) \bar{x}_{t+N \mid t} \in \overline{\mathbb{X}}_{F}$ and, in view of (20), (22) $\Sigma_{t+N+1 \mid t} \preceq \Phi \bar{\Sigma} \Phi^{T}+\Psi \bar{\Omega} \Psi^{T}=\bar{\Sigma}$, hence verifying both (19) and (20) at time $t+1$.

The proof of convergence is partially inspired by [29]. In view of the feasibility, at time $t+1$ of the possibly suboptimal solution $\bar{u}_{t+1, \ldots, t+N \mid t}^{f}, K_{t+1, \ldots, t+N \mid t}^{f}, L_{t+1, \ldots, t+N \mid t}^{f}$, and $\left(\bar{x}_{t+1 \mid t}, \Sigma_{t+1 \mid t}\right)$, we have that the optimal cost function computed at time $t+1$ is $J^{*}(t+1)=J_{m}^{*}(t+1)+$
$J_{v}^{*}(t+1)^{1}$. In view of the optimality of $J^{*}(t+1)$

$$
\begin{aligned}
J^{*}(t+1) & \leq J_{m}\left(\bar{x}_{t+1 \mid t}, \bar{u}_{t+1, \ldots, t+N \mid t}^{f}\right) \\
& +J_{v}\left(\Sigma_{t+1 \mid t}, K_{t+1, \ldots, t+N \mid t}^{f}, L_{t+1, \ldots, t+N \mid t}^{f}\right)
\end{aligned}
$$

Note that, in view of (17)

$$
\begin{aligned}
& J_{m}\left(\bar{x}_{t+1 \mid t}, \bar{u}_{t+1, \ldots, t+N \mid t}^{f}\right) \leq \\
& J_{m}\left(\bar{x}_{t \mid t}, \bar{u}_{t, \ldots, t+N-1 \mid t}\right)-\left\|\bar{x}_{t \mid t}\right\|_{Q}^{2}-\left\|\bar{u}_{t \mid t}\right\|_{R}^{2}
\end{aligned}
$$

Furthermore

$$
J_{m}\left(\bar{x}_{t \mid t}, \bar{u}_{t, \ldots, t+N-1 \mid t}\right)=J_{m}^{*}(t)
$$

Now consider $J_{v}$ in (18) and note that, in view of the properties of the trace and (17)

$$
\begin{aligned}
& J_{v}\left(X_{t+1 \mid t}, K_{t+1, \ldots, t+N \mid t}^{f}, L_{t+1, \ldots, t+N \mid t}^{f}\right) \\
& \leq J_{v}\left(X_{t \mid t}, K_{t, \ldots, t+N-1 \mid t}, L_{t, \ldots, t+N-1 \mid t}\right) \\
& -\operatorname{tr}\left\{\left[\begin{array}{cc}
Q_{L} & 0 \\
0 & Q+K_{t \mid t}^{T} R K_{t \mid t}
\end{array}\right] \Sigma_{t \mid t}\right\}+\operatorname{tr}\left(S_{T} \Psi \Omega \Psi^{T}\right)
\end{aligned}
$$

From (A.1)-(A.4) we obtain

$$
\begin{aligned}
& J^{*}(t+1) \leq J^{*}(t)-\left(\left\|\bar{x}_{t \mid t}\right\|_{Q}^{2}+\left\|\bar{u}_{t \mid t}\right\|_{R}^{2}\right) \\
& -\operatorname{tr}\left\{\left[\begin{array}{cc}
Q_{L} & 0 \\
0 & Q+K_{t \mid t}^{T} R K_{t \mid t}
\end{array}\right] \Sigma_{t \mid t}\right\}+\operatorname{tr}\left(S_{T} \Psi \Omega \Psi^{T}\right)
\end{aligned}
$$

Furthermore, from the definition of $J^{*}(t)$

$$
\begin{aligned}
J^{*}(t) & \geq\left\|\bar{x}_{t \mid t}\right\|_{Q}^{2}+\left\|\bar{u}_{t \mid t}\right\|_{R}^{2} \\
& +\operatorname{tr}\left\{\left[\begin{array}{cc}
Q_{L} & 0 \\
0 & Q+K_{t \mid t}^{T} R K_{t \mid t}
\end{array}\right] \Sigma_{t \mid t}\right\}
\end{aligned}
$$

Now, denote $\Omega_{F}=\left\{(\bar{x}, \Sigma): \bar{x} \in \overline{\mathbb{X}}_{F}, \Sigma \preceq \bar{\Sigma}\right\}$. Assuming that $\left(\bar{x}_{t \mid t}, \Sigma_{t \mid t}\right) \in \Omega_{F}$ we have that $J^{*}(t) \leq J_{m}^{a u x}(t)+$ $J_{v}^{\text {aux }}(t)$, where

$$
\begin{aligned}
J_{m}^{a u x}(t) & =\sum_{k=0}^{N-1}\left\|(A-B \bar{K})^{k} \bar{x}_{t \mid t}\right\|_{Q}^{2} \\
& +\left\|\bar{K}(A-B \bar{K})^{k} \bar{x}_{t \mid t}\right\|_{R}^{2}+\left\|(A-B \bar{K})^{N} \bar{x}_{t \mid t}\right\|_{S}^{2}
\end{aligned}
$$

since $\left\{-\bar{K} \bar{x}_{t \mid t}, \ldots,-\bar{K}(A-B \bar{K})^{N-1} \bar{x}_{t \mid t}\right\}$ is a feasible input sequence. Therefore, from (17),

$$
J_{m}^{a u x}(t) \leq\left\|\bar{x}_{t \mid t}\right\|_{S}^{2}
$$

\footnotetext{
1 For brevity, we denote $J^{*}\left(x_{t}, \bar{x}_{t \mid t-1}, \Sigma_{t \mid t-1}\right)$ with $J^{*}(t)$, $J_{m}^{*}\left(x_{t}, \bar{x}_{t \mid t-1}, \Sigma_{t \mid t-1}\right)$ with $J_{m}^{*}(t)$, and $J_{v}^{*}\left(x_{t}, \bar{x}_{t \mid t-1}, \Sigma_{t \mid t-1}\right)$ with $J_{v}^{*}(t)$
} 
Similarly, recalling (17), we obtain that

$$
J_{v}^{a u x}(t) \leq \operatorname{tr}\left\{S_{T} \Sigma_{t \mid t}\right\}+N \operatorname{tr}\left\{S_{T} \Psi \Omega \Psi^{T}\right\}
$$

Combining (A.7) and (A.8) we obtain that, for all $\left(\bar{x}_{t \mid t}, \Sigma_{t \mid t}\right) \in \Omega_{F}$

$$
J^{*}(t) \leq\left\|\bar{x}_{t \mid t}\right\|_{S}^{2}+\operatorname{tr}\left\{S_{T} \Sigma_{t \mid t}\right\}+N \operatorname{tr}\left\{S_{T} \Psi \Omega \Psi^{T}\right\}
$$

From (A.5), (A.6) and (A.9) it is possible to derive robust stability-related results.

Before to proceed, recall that $\operatorname{tr}\left\{S_{T} \Sigma_{t \mid t}\right\}=\operatorname{tr}\left\{S_{T}^{\frac{1}{2} T} \Sigma_{t \mid t} S_{T}^{\frac{1}{2}}\right\}$ where $S_{T}^{\frac{1}{2}}$ is a matrix that verifies $S_{T}^{\frac{1}{2} T} S_{T}^{\frac{1}{2}}=S_{T}$. Therefore $\operatorname{tr}\left\{S_{T} \Sigma_{t \mid t}\right\}=\operatorname{tr}\left\{S_{T}^{\frac{1}{2} T} \Sigma_{t \mid t} S_{T}^{\frac{1}{2}}\right\}=\left\|\Sigma_{t \mid t}^{\frac{1}{2}} S_{T}^{\frac{1}{2}}\right\|_{F}^{2}$. On the other hand, denoting $Q_{T \mid t}=\operatorname{diag}\left(Q_{L}, Q+K_{t \mid t}^{T} R K_{t \mid t}\right)$, it follows that $\operatorname{tr}\left\{Q_{T \mid t} \Sigma_{t \mid t}\right\}=\left\|\Sigma_{t \mid t}^{\frac{1}{2}} Q_{T \mid t}^{\frac{1}{2}}\right\|_{F}^{2}$. Moreover, $\operatorname{tr}\left\{S_{T} \Sigma_{t \mid t}\right\} \leq\left\|\Sigma_{t \mid t}^{\frac{1}{2}}\right\|_{F}^{2}\left\|S_{T}^{\frac{1}{2}}\right\|_{F}^{2}=\operatorname{tr}\left\{S_{T}\right\} \operatorname{tr}\left\{\Sigma_{t \mid t}\right\}$ (where $\|\cdot\|_{F}$ is the Frobenius norm) and, in view of the matrix inversion Lemma, $\operatorname{tr}\left\{Q_{T \mid t} \Sigma_{t \mid t}\right\} \geq\left(\left\|Q_{T \mid t}^{-\frac{1}{2}}\right\|_{F}^{2}\right)^{-1}\left\|\Sigma_{t \mid t}^{\frac{1}{2}}\right\|_{F}^{2} \geq$ $\operatorname{tr}\left\{\left(\operatorname{diag}\left(Q_{L}, Q\right)\right)^{-1}\right\}^{-1} \operatorname{tr}\left\{\Sigma_{t \mid t}\right\}=\operatorname{tr}\left\{Q^{-1}+Q_{L}^{-1}\right\}^{-1} \operatorname{tr}\left\{\Sigma_{t \mid t}\right\}$. Define $V\left(\bar{x}_{t \mid t}, \Sigma_{t \mid t}\right)=\left\|\bar{x}_{t \mid t}\right\|^{2}+\operatorname{tr}\left\{\Sigma_{t \mid t}\right\}$ and $\omega=$ $\operatorname{tr}\left\{S_{T} \Psi \Omega \Psi^{T}\right\}$. In view of this, we can reformulate (A.5), (A.6) and (A.9) as follows.

$$
\begin{aligned}
J^{*}(t+1) & \leq J^{*}(t)-\alpha V\left(\bar{x}_{t \mid t}, \Sigma_{t \mid t}\right)+\omega \\
J^{*}(t) & \geq \alpha V\left(\bar{x}_{t \mid t}, \Sigma_{t \mid t}\right) \\
J^{*}(t) & \leq \beta V\left(\bar{x}_{t \mid t}, \Sigma_{t \mid t}\right)+N \omega
\end{aligned}
$$

If $\left(\bar{x}_{t \mid t}, \Sigma_{t \mid t}\right) \in \Omega_{F}$ then, in view of (A.10c), (A.10a)

$$
J^{*}(t+1) \leq J^{*}(t)\left(1-\frac{\alpha}{\beta}\right)+\left(\frac{\alpha}{\beta} N+1\right) \omega
$$

Let $\eta \in(\rho, 1)$ and denote $b=\frac{1}{\eta}\left(N+\frac{\beta}{\alpha}\right)$. In view of (A.10b), if $J^{*}(t) \leq b \omega$ then $V\left(\bar{x}_{t \mid t}, \Sigma_{t \mid t}\right) \leq \frac{b}{\alpha} \omega$. This, considering (26), implies that

$$
\left\|\bar{x}_{t \mid t}\right\|^{2} \leq \frac{\rho}{\eta} \bar{\sigma}^{2}, \operatorname{tr}\left(\Sigma_{t \mid t}\right) \leq \frac{\rho}{\eta} \lambda_{\min }(\bar{\Sigma})
$$

In view of (A.12), then $\bar{x}_{t \mid t} \in \overline{\mathbb{X}}_{F}$ and $\lambda_{\max }\left(\Sigma_{t \mid t}\right)<$ $\lambda_{\min }(\bar{\Sigma})$, which in turn implies that $\Sigma_{t \mid t}<\bar{\Sigma}$. Therefore, recalling (A.11), if $J^{*}(t) \leq b \omega$, then $J^{*}(t+1) \leq b \omega$ and the positive invariance of the set $D=\left\{(\bar{x}, \Sigma): J^{*}(t) \leq\right.$ $b \omega\}$ is guaranteed.

If $\left(\bar{x}_{t \mid t}, \Sigma_{t \mid t}\right) \in \Omega_{F} \backslash D$, it holds that $J^{*}(t)>b \omega$ which, in view of $(\mathrm{A} .10 \mathrm{c})$, implies that

$$
V\left(\bar{x}_{t \mid t}, \Sigma_{t \mid t}\right)>\frac{1}{\alpha} \omega
$$

Since $\left(\bar{x}_{t \mid t}, \Sigma_{t \mid t}\right) \in \Omega_{F} \backslash D$, recalling (A.11), (A.13), and (A.10b), there exists $\bar{c}_{1}>0$ (function of $\eta$ ) such that

$$
J^{*}(t+1)-J^{*}(t) \leq-(1-\eta) \frac{\alpha^{2}}{\beta} V\left(\bar{x}_{t \mid t}, \Sigma_{t \mid t}\right) \leq-\bar{c}_{1}
$$

On the other hand, for all $x_{t}$ with $\left(\bar{x}_{t \mid t}, \Sigma_{t \mid t}\right) \in \Xi \backslash \Omega_{F}$, there exists constant $\bar{c}_{2}>0$ such that there exists $x_{\Omega}$ with $\left(\bar{x}_{\Omega}, \Sigma_{\Omega}\right) \in \Omega_{F} \backslash D$ such that $-\alpha V\left(\bar{x}_{t \mid t}, \Sigma_{t \mid t}\right) \leq$ $-\alpha V\left(\bar{x}_{\Omega}, \Sigma_{\Omega}\right)-\bar{c}_{2}$. This, in view of (A.10a) and (A.14), implies that

$$
J^{*}(t+1)-J^{*}(t)<-\bar{c}_{2}
$$

In view of (A.14)-(A.15), for all $x_{t}$ with $\left(\bar{x}_{t \mid t}, \Sigma_{t \mid t}\right) \in$ $\Xi \backslash D$ there exists $\bar{c}$ (function of $\eta$ )

$$
J^{*}(t+1)-J^{*}(t)<-\bar{c}
$$

This implies that, for each $\eta \in(\rho, 1)$, there exists $T>0$ such that $x_{t+T}$ is such that $\left(\bar{x}_{t+T \mid t+T}, \Sigma_{t+T \mid t+T}\right) \in D$, i.e., that $\alpha V\left(\bar{x}_{t+k \mid t+k}, \Sigma_{t+k \mid t+k}\right) \leq b \omega$ for all $k \geq T$. This, for $\eta \rightarrow 1$, implies (28).

\section{B The S-MPCl problem}

\section{B.1 Proof of Lemma 1}

For the proof of Part I., the following result is used.

Lemma 2 Given a positive semi-definite, symmetric matrix $M$, then

$$
M=\left[\begin{array}{cc}
M_{11} & M_{12} \\
M_{12}^{T} & M_{22}
\end{array}\right] \preceq\left[\begin{array}{cc}
2 M_{11} & 0 \\
0 & 2 M_{22}
\end{array}\right]
$$

Proof of Lemma 2 Since $M \succeq 0$, then $\left[\begin{array}{ll}-x_{1}^{T} & x_{2}^{T}\end{array}\right] M\left[\begin{array}{c}-x_{1} \\ x_{2}\end{array}\right]=$ $x_{1}^{T} M_{11} x_{1}+x_{2}^{T} M_{22} x_{2}-x_{1}^{T} M_{12} x_{2}-x_{2}^{T} M_{12}^{T} x_{1} \succeq 0$ for all $x_{1}, x_{2}$ such that $\left[x_{1}^{T} x_{2}^{T}\right] \neq 0$. From this, we obtain that $\left[x_{1}^{T} x_{2}^{T}\right] M\left[\begin{array}{l}x_{1} \\ x_{2}\end{array}\right]=x_{1}^{T} M_{11} x_{1}+x_{2}^{T} M_{22} x_{2}+$ $x_{1}^{T} M_{12} x_{2}+x_{2}^{T} M_{12}^{T} x_{1} \leq 2 x_{1}^{T} M_{11} x_{1}+2 x_{2}^{T} M_{22} x_{2}=$ $\left[\begin{array}{ll}x_{1}^{T} & x_{2}^{T}\end{array}\right]\left[\begin{array}{cc}2 M_{11} & 0 \\ 0 & 2 M_{22}\end{array}\right]\left[\begin{array}{l}x_{1} \\ x_{2}\end{array}\right]$ for all $x_{1}, x_{2}$ such that $\left[\begin{array}{ll}x_{1}^{T} & x_{2}^{T}\end{array}\right] \neq 0$. This concludes the proof of Lemma 2 . Consider now matrix $\Sigma_{t}$ and its block decomposition $\Sigma_{t}=\left[\begin{array}{ll}\Sigma_{11, t} & \Sigma_{12, t} \\ \Sigma_{12, t}^{T} & \Sigma_{22, t}\end{array}\right]$ 
where $\Sigma_{i j, t} \in \mathbb{R}^{n \times n}$ for all $i, j=1,2$. A bound for the time evolution of the covariance matrix $\Sigma_{t}$ is computed, iteratively, considering that

$$
\Sigma_{t+1} \preceq \Phi_{t} \Sigma_{t}^{D} \Phi_{t}^{T}+\Psi_{t} \Omega \Psi_{t}^{T}
$$

If we define $\Sigma_{t+1}^{D}=\operatorname{diag}\left(\Sigma_{11, t+1}^{D}, \Sigma_{22, t+1}^{D}\right)$, where

$$
\begin{gathered}
\Sigma_{11, t+1}^{D}=2\left(A-L_{t} C\right) \Sigma_{11, t}^{D}\left(A-L_{t} C\right)^{T}+ \\
F W F^{T}+2 L_{t} V L_{t}^{T} \\
\Sigma_{22, t+1}^{D}=2\left(A-B K_{t}\right) \Sigma_{22, t}^{D}\left(A-B K_{t}\right)^{T}+ \\
2 L_{t} C \Sigma_{11, t}^{D} C^{T} L_{t}^{T}+2 L_{t} V L_{t}^{T}
\end{gathered}
$$

then we obtain that $\Sigma_{t+1}^{D} \succeq \Sigma_{t+1}$, in view of Lemma 2 . The latter corresponds with (29).

\section{Part IIa. LMI reformulation of the update of $\Sigma_{11, k}^{D}$.}

We define $\Gamma_{k}=\operatorname{diag}\left(\Sigma_{11, k}^{D}, W^{-1},\left(V^{D}\right)^{-1}\right), \quad \tilde{\Theta}_{k}=$ $\left[\begin{array}{lll}A_{L_{k}}^{D} & F W & L_{k} V^{D}\end{array}\right]$ and we rewrite constraint (30a) as $\Sigma_{11, k+1}^{D}-\tilde{\Theta}_{k} \Gamma_{k} \tilde{\Theta}_{k}^{T} \succeq 0$. Resorting to the Schur complement it is possible to derive the equivalent form $\Gamma_{k}^{-1}-\tilde{\Theta}_{k}^{T}\left(\Sigma_{11, k+1}^{D}\right)^{-1} \tilde{\Theta}_{k} \succeq 0$. To obtain a linear inequality from the previous expression we define

$$
Z_{k}=\left(\Sigma_{11, k+1}^{D}\right)^{-1} L_{k}
$$

and $\tilde{\Sigma}_{11, i}^{D}=\left(\Sigma_{11, i}^{D}\right)^{-1}$, i.e., $\operatorname{diag}\left(\tilde{\Sigma}_{11, k}^{D}, W, V^{D}\right)-$ $\Phi_{k}^{T}\left(\tilde{\Sigma}_{11, k+1}^{D}\right)^{-1} \Phi_{k} \succeq 0$, where $\Phi_{k}=\left[\left(\tilde{\Sigma}_{11, k+1}^{D} A^{D}-\right.\right.$ $\left.\left.Z_{k} C^{D}\right), \tilde{\Sigma}_{11, k+1}^{D} F W, Z_{k} V^{D}\right]$. The latter expression can be written as a compact LMI as follows

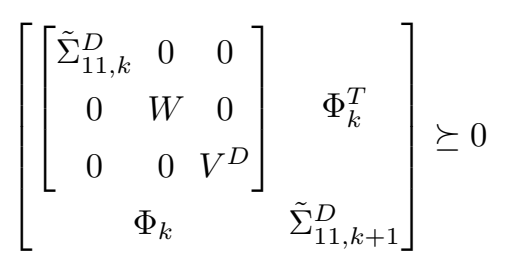

Notice that, however, in the constraints (32) and in the cost function (36), the term $\Sigma_{11, i}^{D}$ appears, rather than its inverse $\tilde{\Sigma}_{11, i}^{D}$. To solve this issue, we define matrix $\Delta_{k}$ as an upper bound to $\Sigma_{11, k}^{D}$ (i.e., $\Delta_{k} \succeq \Sigma_{11, k}^{D}$ ), which can be recovered from $\tilde{\Sigma}_{11, k+1}^{D}$ through the following linear inequality

$$
\left[\begin{array}{cc}
\Delta_{k} & I \\
I & \tilde{\Sigma}_{11, k}^{D}
\end{array}\right] \succeq 0
$$

Then, one should replace $\Sigma_{11, k}^{D}$ with $\Delta_{k}$ in (32) and (36).

Part IIb. Reformulation of the update of $\Sigma_{22, k}^{D}$.
Consider now the inequality (30b), i.e.,

$$
\begin{aligned}
& \Sigma_{22, k+1}^{D}-\left(A^{D}-B^{D} K_{k}\right) \Sigma_{22, k}^{D}\left(A^{D}-B^{D} K_{k}\right)^{T} \\
& -L_{k}\left(C^{D} \Sigma_{11, k}^{D} C^{D T}+V^{D}\right) L_{k}^{T} \succeq 0
\end{aligned}
$$

Recalling (B.4), (B.7) can be rewritten as $\Sigma_{22, k+1}^{D}-$ $\left(A^{D}-B^{D} K_{k}\right) \Sigma_{22, k}^{D}\left(A^{D}-B^{D} K_{k}\right)^{T}-\Sigma_{11, k+1}^{D} M_{k} \Sigma_{11, k+1}^{D} \succeq 0$, where

$$
M_{k}=Z_{k}\left(C^{D} \Sigma_{11, k}^{D} C^{D T}+V^{D}\right) Z_{k}^{T}
$$

By defining $\Xi_{k}=K_{k} \Sigma_{22, k}^{D}$, and using the matrix $\Delta_{k+1}$ in place of $\Sigma_{11, k+1}^{D}$, the inequality (B.7) can be recast as a suitable LMI. In fact, in view of the Schur complement Lemma and letting $\tilde{M}_{k}=M_{k}^{-1}$, we obtain

$$
\left[\begin{array}{c}
\Sigma_{22, k+1}^{D} \\
{\left[\begin{array}{c}
\left(A^{D} \Sigma_{22, k}^{D}-B^{D} \Xi_{k}\right)^{T} \\
\Delta_{k+1}
\end{array}\right]} \\
{\left[\left(A^{D} \Sigma_{22, k}^{D}-B^{D} \Xi_{k}\right) \Delta_{k+1}\right.} \\
{\left[\begin{array}{cc}
\Sigma_{22, k}^{D} & 0 \\
0 & \tilde{M}_{k}
\end{array}\right]}
\end{array}\right] \succeq 0
$$

The equation (B.8) can be recast as the inequality $M_{k} \succeq$ $Z_{k}\left(C^{D} \Sigma_{11, k}^{D} C^{D T}+V^{D}\right) Z_{k}^{T}$, which can be reformulated as

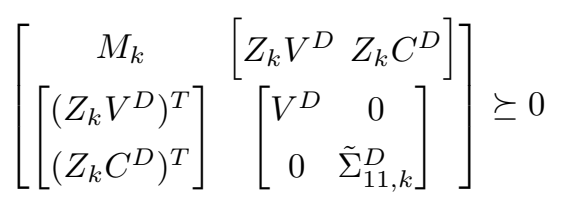

Finally, concerning the equality $\tilde{M}_{k}=M_{k}^{-1}$, it can be solved using the approach proposed in [7]. Indeed, we solve the following LMI

$$
\left[\begin{array}{cc}
M_{k} & I \\
I & \tilde{M}_{k}
\end{array}\right] \succeq 0
$$

and, at the same time, we minimize the additional cost function

$$
\operatorname{tr}\left\{M_{k} \tilde{M}_{k}\right\}
$$

The problem (B.11)-(B.12) can be managed using the recursive cone complementarity linearization algorithm proposed in [7] with a suitable initialization.

\section{B.2 LMI reformulation of the constraints}

While the constraint (31a) is a linear inequality (and therefore it does not need to be further reformulated), the inequality (31b) needs special attention. As already remarked, in (31b), $U_{k}$ must be replaced by $\bar{U}_{k}$. In turn, the equality $\bar{U}_{k}=K_{k} \Sigma_{22, k}^{D} K_{k}^{T}=\Xi_{k}\left(\Sigma_{22, k}^{D}\right)^{-1} \Xi_{k}^{T}$ must be recast as an LMI as follows:

$$
\left[\begin{array}{cc}
\bar{U}_{k} & \Xi_{k} \\
\Xi_{k}^{T} & \Sigma_{22, k}^{D}
\end{array}\right] \succeq 0
$$




\section{References}

[1] D. Bernardini and A. Bemporad. Stabilizing model predictive control of stochastic constrained linear systems. IEEE Transactions on Automatic Control, 57(6):1468-1480, 2012.

[2] M. Bichi, G. Ripaccioli, S. Di Cairano, D. Bernardini, A. Bemporad, and I.V. Kolmanovsky. Stochastic model predictive control with driver behavior learning for improved powertrain control. In IEEE Conference on Decision and Control (CDC), pages 6077-6082, 2010.

[3] L. Blackmore, M. Ono, A. Bektassov, and B. C. Williams. A probabilistic particle-control approximation of chanceconstrained stochastic predictive control. IEEE Transactions on Robotics, 26:502-517, 2010.

[4] G. C. Calafiore and L. Fagiano. Robust model predictive control via scenario optimization. IEEE Transactions on Automatic Control, 58:219-224, 2013.

[5] M. Cannon, Q. Cheng, B. Kouvaritakis, and S. V. Raković. Stochastic tube MPC with state estimation. Automatica, 48(3):536 - 541, 2012.

[6] M. Cannon, B. Kouvaritakis, and X. Wu. Model predictive control for systems with stochastic multiplicative uncertainty and probabilistic constraints. Automatica, 45(1):167 - 172, 2009.

[7] L. El Ghaoui, F. Oustry, and M. AitRami. A cone complementarity linearization algorithm for static outputfeedback and related problems. IEEE Transactions on Automatic Control, 42(8):1171-1176, 1997.

[8] M. Farina, L. Giulioni, L. Magni, and R. Scattolini. A probabilistic approach to model predictive control. In Proceedings of the IEEE Conference on Decision and Control, pages 7734-7739, 2013.

[9] M. Farina, L. Giulioni, L. Magni, and R. Scattolini. An mpc approach to output-feedback control of stochastic linear discrete-time systems. In IFAC World Congress, pages 74617466,2014

[10] M. Farina, L. Giulioni, L. Magni, and R. Scattolini. An MPC approach to output-feedback control of stochastic linear discrete-time systems. arXiv:1408.6723, available at http://arxiv.org/abs/1408.6723, 2014.

[11] T. Glad and L. Ljung. Control theory. CRC press, 2000.

[12] A. Gray, Yiqi Gao, T. Lin, J.K. Hedrick, and F. Borrelli. Stochastic predictive control for semi-autonomous vehicles with an uncertain driver model. In International IEEE Conference on Intelligent Transportation Systems (ITSC), pages 2329-2334, 2013.

[13] P. Hokayem, E. Cinquemani, D. Chatterjee, F. Ramponi, and J. Lygeros. Stochastic receding horizon control with output feedback and bounded controls. Automatica, 48:77-88, 2012.

[14] J. Hu, Z. Wang, H. Gao, and L. K. Stergioulas. Probabilityguaranteed $\mathrm{H}_{\infty}$ finite-horizon filtering for a class of nonlinear time-varying systems with sensor saturations. Systems 8 Control Letters, 61(4):477 - 484, 2012.

[15] M. Korda, R. Gondhalekar, F. Oldewurtel, and C.N. Jones. Stochastic MPC framework for controlling the average constraint violation. IEEE Transactions on Automatic Control, 59(7):1706-1721, 2014.

[16] B. Kouvaritakis, M. Cannon, S. V. Rakovic, and Q. Cheng. Explicit use of probabilistic distributions in linear predictive control. Automatica, 46(10):1719 - 1724, 2010.

[17] P. Li, M. Wendt, and G. Wozny. A probabilistically constrained model predictive controller. Automatica, 38:1171-1176, 2002.
[18] D. Limon, T. Alamo, D. M. Raimondo, D. Mu noz de la Peña, J. M. Bravo, A. Ferramosca, and E. F. Camacho. Inputto-state stability: an unifying framework for robust model predictive control. In Nonlinear Model Predictive Control, LNCIS 384, pages 1-26, 2009.

[19] A. Locatelli, F. Romeo, and R. Scattolini. On the design of optimal decentralized controllers under control path constraints. Large Scale Systems, 5(3):221-231, 1983.

[20] L. Magni, G. De Nicolao, R. Scattolini, and F. Allgöwer. Robust model predictive control for nonlinear discrete-time systems. International Journal of Robust and Nonlinear Control, 13(3-4):229-246, 2003.

[21] L. Magni, D. Pala, and R. Scattolini. Stochastic model predictive control of constrained linear systems with additive uncertainty. Proceedings of the European Control Conference, Budapest, Hungary, pages 2235-2240, 2009.

[22] A. W. Marshall and I. Olkin. Multivariate Chebychev inequalities. Annals of Mathematical Statistics, 34(4):10011014, 1960 .

[23] D. Q. Mayne, J. B. Rawlings, C. V. Rao, and P. O. M. Scokaert. Constrained model predictive control: Stability and optimality. Automatica, 36:789-814, 2000.

[24] D.Q. Mayne, S.V. Raković, R. Findeisen, and F. Allgöwer. Robust output feedback model predictive control of constrained linear systems. Automatica, 42(7):1217 - 1222, 2006.

[25] D.Q. Mayne, M.M. Seron, and S.V. Rakovic. Robust model predictive control of constrained linear systems with bounded disturbances. Automatica, 41(2):219 - 224, 2005.

[26] F. Oldewurtel, C.N. Jones, A Parisio, and M. Morari. Stochastic model predictive control for building climate control. IEEE Transactions on Control Systems Technology, 22(3):1198-1205, 2014.

[27] M. Ono. Joint chance-constrained model predictive control with probabilistic resolvability. In American Control Conference (ACC), 2012, pages 435-441, 2012.

[28] J. A. Primbs and C. H. Sung. Stochastic receding horizon control of constrained linear systems with state and control multiplicative noise. IEEE Transactions on Automatic Control, 54(2):221-230, 2007.

[29] D. M. Raimondo, D. Limon, M. Lazar, L. Magni, and E. F. Camacho. Min-max model predictive control of nonlinear systems: A unifying overview on stability. European Journal of Control, 15:5-21, 2009.

[30] J.B. Rawlings and D.Q. Mayne. Model Predictive Control: Theory and Design. Nob Hill Publishing, 2009.

[31] A.T. Schawarm and M. Nikolaou. Chance-constrained model predictive control. AIChE Journal, 45(8):1743-1752, 1999.

[32] I. Yaesh, S. Boyarski, and U. Shaked. Probability-guaranteed robust $\mathrm{H}_{\infty}$ performance analysis and state-feedback design. Systems \& Control Letters, 48(5):351 - 364, 2003.

[33] J. Yan and R. R. Bitmead. Incorporating state estimation into model predictive control and its application to network traffic control. Automatica, 41:595-604, 2005.

[34] Z. Zhou and R. Cogill. Reliable approximations of probability-constrained stochastic linear-quadratic control. Automatica, 49:2435-2439, 2013. 